\title{
Ceftriaxone to PRevent pneumOnia and inflammaTion aftEr Cardiac arresT (PROTECT): study protocol for a randomized, placebo-controlled trial
}

David J. Gagnon 1,2,3* , Sergey V. Ryzhov², Meghan A. May ${ }^{4}$, Richard R. Riker ${ }^{3,5}$, Bram Geller ${ }^{3,6}$, Teresa L. May 2,3,5, Sarah Bockian? ${ }^{7}$, Joanne T. deKay ${ }^{2}$, Ashley Eldridge ${ }^{7}$, Thomas Van der Kloot ${ }^{5}$, Patricia Lerwick ${ }^{5}$, Christine Lord ${ }^{7}$, F. Lee Lucas ${ }^{2}$, Patrick Mailloux ${ }^{5}$, Barbara McCrum ${ }^{7}$, Meghan Searight ${ }^{7}$, Joel Wirth ${ }^{5}$, Jonathan Zuckerman ${ }^{3}$, Douglas Sawyer 2,6 and David B. Seder 2,3,5

\begin{abstract}
Background: Pneumonia is the most common infection after out-of-hospital cardiac arrest (OHCA) occurring in up to $65 \%$ of patients who remain comatose after return of spontaneous circulation. Preventing infection after OHCA may (1) reduce exposure to broad-spectrum antibiotics, (2) prevent hemodynamic derangements due to local and systemic inflammation, and (3) prevent infection-associated morbidity and mortality.

Methods: The ceftriaxone to PRevent pneumOnia and inflammaTion aftEr Cardiac arrest (PROTECT) trial is a randomized, placebo-controlled, single-center, quadruple-blind (patient, treatment team, research team, outcome assessors), noncommercial, superiority trial to be conducted at Maine Medical Center in Portland, Maine, USA. Ceftriaxone $2 \mathrm{~g}$ intravenously every $12 \mathrm{~h}$ for 3 days will be compared with matching placebo. The primary efficacy outcome is incidence of early-onset pneumonia occurring $<4$ days after mechanical ventilation initiation. Concurrently, T cell-mediated inflammation bacterial resistomes will be examined. Safety outcomes include incidence of type-one immediate-type hypersensitivity reactions, gallbladder injury, and Clostridioides difficile-associated diarrhea. The trial will enroll 120 subjects over approximately 3 to 4 years.

Discussion: The PROTECT trial is novel in its (1) inclusion of OHCA survivors regardless of initial heart rhythm, (2) use of a low-risk antibiotic available in the USA that has not previously been tested after OHCA, (3) inclusion of anti-inflammatory effects of ceftriaxone as a novel mechanism for improved clinical outcomes, and (4) complete metagenomic assessment of bacterial resistomes pre- and post-ceftriaxone prophylaxis. The long-term goal is to develop a definitive phase III trial powered for mortality or functional outcome.
\end{abstract}

Trial registration: ClinicalTrials.gov NCT04999592. Registered on August 10, 2021.

Keywords: Cardiac arrest, Pneumonia, Targeted temperature management, Hypothermia, Antibiotics, Inflammation, Microbiome, Metagenomics, Ceftriaxone

\footnotetext{
* Correspondence: dgagnon@mmc.org

'Department of Pharmacy, Maine Medical Center, Portland, ME, USA

${ }^{2}$ Maine Medical Center Research Institute, Scarborough, ME, USA

Full list of author information is available at the end of the article
}

C The Author(s). 2022 Open Access This article is licensed under a Creative Commons Attribution 4.0 International License, which permits use, sharing, adaptation, distribution and reproduction in any medium or format, as long as you give appropriate credit to the original author(s) and the source, provide a link to the Creative Commons licence, and indicate if changes were made. The images or other third party material in this article are included in the article's Creative Commons licence, unless indicated otherwise in a credit line to the material. If material is not included in the article's Creative Commons licence and your intended use is not permitted by statutory regulation or exceeds the permitted use, you will need to obtain permission directly from the copyright holder. To view a copy of this licence, visit http://creativecommons.org/licenses/by/4.0/ The Creative Commons Public Domain Dedication waiver (http://creativecommons.org/publicdomain/zero/1.0/) applies to the data made available in this article, unless otherwise stated in a credit line to the data. 


\section{Administrative information}

Note: the numbers in curly brackets in this protocol refer to SPIRIT checklist item numbers. The order of the items has been modified to group similar items (see http://www.equator-network.org/reporting-guidelines/ spirit-2013-statement-defining-standard-protocol-itemsfor-clinical-trials/).

\begin{tabular}{ll}
\hline Title $\{1\} \quad$ & ceftriaxone to PRevent pneumOnia and \\
& inflammaTion aftEr Cardiac arresT \\
& (PROTECT): a randomized-controlled \\
& trial and microbiome assessment
\end{tabular}

Trial registration $\{2 \mathrm{a}$ and $2 \mathrm{~b}\}$. ClinicalTrials.gov (NCT04999592)

registered August 10, 2021

Protocol version $\{3\}$

Funding $\{4\}$

Author details $\{5 \mathrm{a}\}$ trial protocol version 3 dated 6/11/21.

The trial is supported by the National Institute of General Medical Sciences (1P20GM139745-01).

David J. Gagnon
This manuscript describes the PROTECT

- Department of Pharmacy, Maine Medical Center, Portland, ME, USA

- Maine Medical Center Research Institute, Scarborough, ME, USA

- Tufts University School of Medicine, Boston, MA, USA

Sergey V. Ryzhov

- Maine Medical Center Research Institute, Scarborough, ME, USA Meghan A. May

- University of New England College of Osteopathic Medicine, Biddeford, ME, USA

Richard R. Riker

- Tufts University School of Medicine,

Boston, MA, USA

- Department of Critical Care Services, Maine Medical Center, Portland, ME, USA

Bram Geller

- Tufts University School of Medicine, Boston, MA, USA

- Maine Medical Partners, MaineHealth Cardiology, Scarborough, ME, USA

Teresa L. May

- Maine Medical Center Research

Institute, Scarborough, ME, USA

- Tufts University School of Medicine, Boston, MA, USA

- Department of Critical Care Services, Maine Medical Center, Portland, ME, USA

Sarah Bockian

- Maine Medical Center Neuroscience Institute, Maine Medical Center, Portland, ME, USA

Joanne T. deKay

- Maine Medical Center Research Institute, Scarborough, ME, USA

Ashley Eldridge

- Maine Medical Center Neuroscience Institute, Maine Medical Center, Portland, ME, USA

Thomas Van der Kloot

- Department of Critical Care Services, Maine Medical Center, Portland, ME,

\section{Administrative information (Continued)}

USA

Patricia Lerwick

- Department of Critical Care Services, Maine Medical Center, Portland, ME, USA

Christine Lord

- Maine Medical Center Neuroscience Institute, Maine Medical Center,

Portland, ME, USA

F. Lee Lucas

- Maine Medical Center Research Institute, Scarborough, ME, USA

Patrick Mailloux

- Department of Critical Care Services, Maine Medical Center, Portland, ME, USA

Barbara McCrum

- Maine Medical Center Neuroscience Institute, Maine Medical Center, Portland, ME, USA

Meghan Searight

- Maine Medical Center Neuroscience Institute, Maine Medical Center,

Portland, ME, USA

Joel Wirth

- Department of Critical Care Services, Maine Medical Center, Portland, ME, USA

Jonathan Zuckerman

- Department of Critical Care Services, Maine Medical Center, Portland, ME, USA

Douglas Sawyer

- Maine Medical Center Research Institute, Scarborough, ME, USA

- Maine Medical Partners, MaineHealth Cardiology, Scarborough, ME, USA

David B. Seder

- Maine Medical Center Research Institute, Scarborough, ME, USA

- Tufts University School of Medicine, Boston, MA, USA

- Department of Critical Care Services, Maine Medical Center, Portland, ME, USA

Name and contact information for the trial National Institute of General Medical Sciences

45 Center Drive MSC 6200

Bethesda, MD 20892-6200

Phone: 301-496-7301

Email: info@nigms.nih.gov

Role of sponsor $\{5 c\}$

The funding agency was not involved with study design, data collection, analysis and interpretation of data, writing of the manuscript, or the decision to submit the manuscript for publication.

\section{Background and rationale $\{6 a\}$}

Pneumonia results in alveolar inflammation and fluid or purulent material accumulation in the lungs [1]. It is the most common infection after cardiac arrest, occurring in up to $65 \%$ of patients treated with targeted temperature management (TTM) [2]. Infections are associated with 
increased intensive care unit (ICU) length of stay (LOS), hospital LOS, duration of mechanical ventilation, postdischarge rehabilitation need, tracheostomy need, and mortality, while also reducing the incidence of a good functional outcome [3-7].

Pneumonia likely results from aspiration during cardiopulmonary resuscitation (CPR) or by introduction of oropharyngeal flora into the lungs during airway management. It may also be due to gastrointestinal hypoperfusion, which leads to ischemic injury to the intestinal mucosa, bacterial translocation, and hematogenous spread of bacteria [8-13]. Infection might also be the result of post-resuscitation immune suppression, but this requires confirmation [14].

Preventing early pneumonia may (1) reduce exposure to broad-spectrum antibiotics and subsequent collateral damage, (2) prevent hemodynamic derangements due to local and systemic inflammation, and (3) prevent an association between infection and morbidity and mortality. These benefits must be carefully balanced with the risk for altering bacterial resistomes, genetic material producing resistance, in the absence of clinical infection.

\section{Objectives $\{7\}$}

The primary objective is to determine if prophylactic ceftriaxone administered within $6 \mathrm{~h}$ of ICU admission reduces the incidence of early-onset pneumonia (EOP). The secondary objectives are to quantify $\mathrm{T}$ cell-mediated inflammation and bacterial resistomes as assessed by resistance genotypes in stool and sputum.

\section{Trial design $\{\mathbf{8}\}$}

The ceftriaxone to PRevent pneumOnia and inflammaTion aftEr Cardiac arresT (PROTECT) is a randomized, placebocontrolled, single-center, quadruple-blind (patient, treatment team, research team, outcome assessors), non-commercial, superiority trial.

\section{Study setting $\{9\}$}

The trial will be conducted at Maine Medical Center in Portland, Maine, USA. The hospital has 637 licensed beds including a 12-bed cardiac ICU and 32-bed mixed medical, surgical, and neurological ICU. It is affiliated with Tufts University School of Medicine in Boston, Massachusetts, USA. Maine Medical Center has managed over 1000 patients with TTM after OHCA and it is the largest hospital in Northern New England. All OHCA patients are admitted to or co-managed by the Neurocritical Care service. The Neurocritical Care team has around-the-clock coverage by an attending physician and an advanced practice provider (i.e., Physician Assistant or Nurse Practitioner).

\section{Eligibility criteria $\{10\}$}

Inclusion criteria:

- $\geq 18$ years of age

- Comatose (do not follow simple verbal commands)

- Have any initial heart rhythm (shockable or nonshockable)

- Out-of-hospital cardiac arrest (OHCA) including the emergency department

Exclusion criteria:

- Name on the Exception from Informed Consent (EFIC) opt-out list

- In-hospital cardiac arrest

- Interval $>6 \mathrm{~h}$ from ICU admission to study drug initiation

- Preexisting terminal disease making 180-day survival unlikely

- Legally authorized representative (LAR) refused informed consent

- Emergent coronary artery bypass grafting

- Anaphylaxis or angioedema to beta-lactam antibiotics (i.e., cephalosporins or penicillins)

- Beta-lactam allergies listed without a known reaction will not be an exclusion

- Under legal guardianship or prisoner

- Known colonization with methicillin-resistant Staphylococcus aureus (MRSA)

- Clinical bacterial infection prior to hospital admission defined as any one of the following:

- Infectious prodrome preceding OHCA

- Active course of antibiotics for infection prior to admission

- Active infection documented in the electronic medical record

- Family or surrogate endorsement of an active infection

- Active course of antibiotics for infectious or noninfectious indications

- Clinical indication for antibiotics at the time of screening in the opinion of the treatment team

\section{Inclusion window}

Controlled studies in heterogeneous cohorts of acutely braininjured patients used four or $6 \mathrm{~h}$ from intubation or $6 \mathrm{~h}$ from ICU admission as the prophylaxis window [15-17]. The Antibiotherapy during Therapeutic Hypothermia to Prevent Infectious Complications (ANTHARTIC) trial, which included only OHCA patients with shockable rhythms, used < $6 \mathrm{~h}$ from return of spontaneous circulation (ROSC) to randomization [18]. Consistent with prior studies, patients will be enrolled in the PROTECT trial if $\leq 6 \mathrm{~h}$ has passed from time of ICU admission to study drug initiation. 
Who will take informed consent? $\{26 \mathrm{a}\}$

Study investigators will approach the patient's Power of Attorney for Health Care (POAHC) or Legally Authorized Representative (LAR) to provide informed consent. Patients regaining consciousness will be asked for informed consent as soon as they have the capacity to do so. On the consent form, subjects will be asked if they agree to use of their data should they choose to withdraw from the trial. Subjects will also be asked for permission for the research team to share relevant data with people from the center, institute, and university taking part in the research, or from regulatory authorities, where relevant.

In the event a patient's POAHC or LAR cannot be reached for informed consent within $30 \mathrm{~min}$, the EFIC process will be initiated per the Food and Drug Administration Guidance for Institutional Review Boards, Clinical Investigators, and Sponsors on Exception from Informed Consent Requirements for Emergency Research [19]. As soon as a POAHC or LAR is found they will be informed of the trial and consented. After community consultation and public disclosure, the Institutional Review Board (IRB) at Maine Medical Center deemed it was appropriate to conduct the trial.

\section{Additional consent provisions for collection and use of participant data and biological specimens $\{26 \mathrm{~b}\}$}

This trial involves collecting biological blood and sputum samples. In the informed consent form, participants are asked whether they agree to the use of their data and biological specimens for future studies.

\section{Interventions}

Explanation for the choice of comparators $\{6 \mathrm{~b}\}$

Ceftriaxone was selected for many reasons: (1) bactericidal activity against commonly isolated bacteria in comatose OHCA patients, (2) generic availability and low cost, (3) ease of administration over $30 \mathrm{~min}$, (4) favorable local susceptibility profile, (5) excellent safety data, and (6) potential neuro-protective effects [20]. Data for bacterial distribution in TTM-1, ANTHARTIC, and local susceptibility to ceftriaxone appear in Table $1[18$, 21]. Bacterial distribution was not reported in the original TTM-2 trial publication [22].

\section{Intervention description $\{11 \mathrm{a}\}$}

Ceftriaxone for injection is a sterile, semi-synthetic, broad-spectrum cephalosporin antibiotic available for intravenous (IV) or intramuscular injection [20]. The ceftriaxone dose of $2 \mathrm{~g}$ IV every $12 \mathrm{~h}$ was selected using internal antibiogram data at Maine Medical Center, the cumulative fraction of response based on dose for methicillin-sensitive Staphylococcus aureus, and preliminary data suggesting it has anti-inflammatory properties (Fig. 1 and Table 2).

Matching placebo of $50 \mathrm{~mL} 0.9 \%$ sodium chloride will be dispensed for patients randomized to the control arm. In order to maintain blinding, an opaque cover will

Table 1 Bacteria identified in the TTM-1 and ANTHARTIC trials with local susceptibility data to ceftriaxone

\begin{tabular}{|c|c|c|c|}
\hline Bacteria & TTM-1 trial ${ }^{\mathrm{a}}$ & 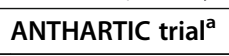 & MMC ceftriaxone susceptibility ${ }^{\mathbf{b}}$ \\
\hline \multicolumn{4}{|l|}{ Gram-positive } \\
\hline Staphylococcus aureus & $22.9 \%$ & $12 \%$ & $86 \%$ \\
\hline Streptococcus pneumoniae & $5.5 \%$ & $7 \%$ & $99 \%$ \\
\hline Streptococcus agalactiae & $1.5 \%$ & $3 \%$ & Not reported \\
\hline \multicolumn{4}{|l|}{ Gram-negative } \\
\hline Haemophilus influenzae & $9.1 \%$ & $22 \%$ & $100 \%$ \\
\hline Escherichia coli & $9.1 \%$ & $11 \%$ & $93 \%$ \\
\hline Klebsiella pneumoniae & $5.1 \%$ & $4 \%$ & $94 \%$ \\
\hline Serratia marcescens & $5.1 \%$ & $3 \%$ & $89 \%$ \\
\hline Klebsiella oxytoca & $3.6 \%$ & $1 \%$ & $97 \%$ \\
\hline Enterobacter cloacae & $3.3 \%$ & $3 \%$ & $78 \%$ \\
\hline Pseudomonas aeruginosa & $2.5 \%$ & $3 \%$ & Not active \\
\hline Enterobacter aerogenes & $2.2 \%$ & $2 \%$ & $75 \%$ \\
\hline Proteus mirabilis & $2.2 \%$ & $1 \%$ & $98 \%$ \\
\hline Moraxella catarrhalis & $1.5 \%$ & $1 \%$ & Not reported \\
\hline
\end{tabular}

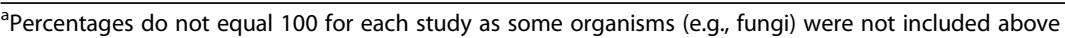

${ }^{b}$ Maine Medical Center ceftriaxone antibiogram data through December 2019

MMC Maine Medical Center, TTM targeted temperature management 


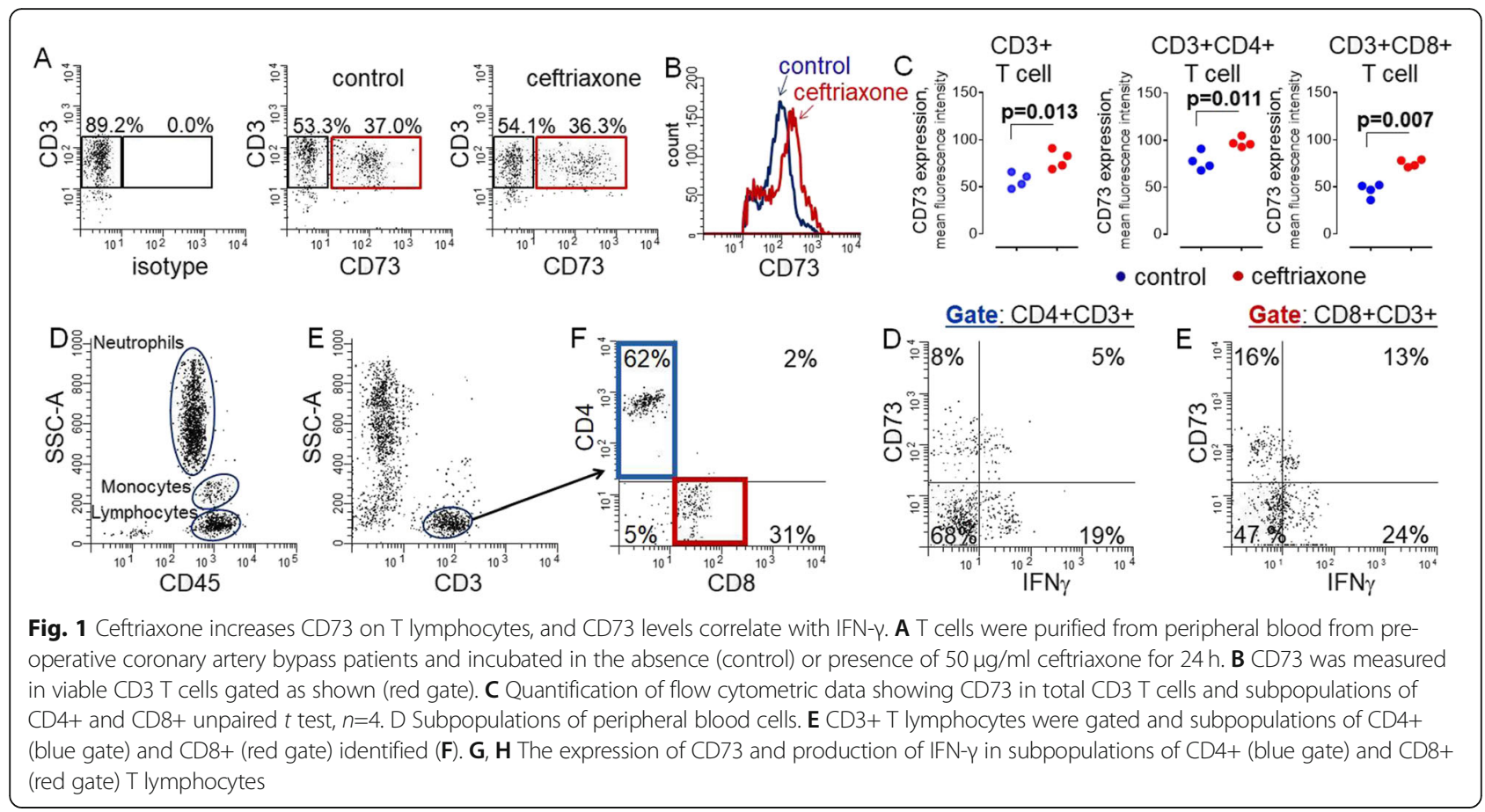

be placed over the bag due to ceftriaxone's light yellow to amber color.

\section{Criteria for discontinuing or modifying allocated interventions $\{11 \mathrm{~b}\}$}

Study drug will be discontinued if:

- Gallbladder toxicity occurs OR;

- Withdrawal from the trial is in the subject's best interest OR;

- Infection develops warranting a change in the antibiotic regimen OR;

- Subject, POAHC, or LAR declines or revokes informed consent.

In the event of stopping the study drug, patients will continue to be followed for safety and efficacy outcomes, but will not have blood, sputum culture, or rectal samples for the inflammation and microbiome assessments.

\section{Strategies to improve adherence to interventions $\{11 \mathrm{c}\}$}

A clinical research associate appointed by Maine Medical Center's Institutional Review Board (IRB) may

Table 2 Cumulative fraction of response to ceftriaxone at various doses for methicillin-sensitive Staphylococcus aureus

\begin{tabular}{ll}
\hline Regimen & Cumulative fraction of response \\
\hline Ceftriaxone 1 g IV q24h & $8 \%$ \\
Ceftriaxone 2 g IV q24h & $45 \%$ \\
Ceftriaxone 2 g IV q12h & $86 \%$ \\
\hline
\end{tabular}

visit during the study to ensure proper conduct. Items for review may include:

- Informed consent forms and EFIC documentation

- Compliance with the study protocol and procedures

- Quality of data collected in the case report form

- Accuracy

- Missing data

- Consistency of the data with the source documents

- Management of the study drug

- Each visit will be recorded in a written monitoring report

\section{Relevant concomitant care permitted or prohibited during the trial $\{11 \mathrm{~d}\}$}

Patients will receive early coronary catheterization, when appropriate, and will undergo multimodal delayed neurologic prognostication according to clinical practice guidelines. All care will be the same in each study group except for the study drug.

Patients unable to follow simple verbal commands after ROSC will be treated with TTM. Patients will be sedated and may receive a dose of a paralytic or chilled saline $\left(4^{\circ} \mathrm{C}\right)$ and cooled to a target temperature per the bedside treatment team $\left(33^{\circ} \mathrm{C}\right.$ to $\left.37.5^{\circ} \mathrm{C}\right)$. The Bard Medical Arctic Sun Temperature Management System, a servo-controlled surface cooling device, will be used.

Propofol is preferred and is titrated by bedside nurses. Analgesia is provided with intermittent injections of fentanyl or a continuous infusion. As needed doses of 
midazolam may be administered. Low doses are maintained to avoid accumulation in the setting of endorgan dysfunction and temperature-mediated decreases in drug metabolism and elimination.

Patients may be monitored with cEEG until they have awoken or it is deemed not clinically necessary. Tracings are read by epileptologists throughout the day and concerning findings are reported to the bedside treatment team. Seizures and epileptiform activity will be classified according to the International League Against Epilepsy, Neurocritical Care Society, and American Clinical Neurophysiology Society's criteria. Antiepiletpic medications may be administered.

Clinical practice guidelines suggest keeping the MAP $>65$ $\mathrm{mmHg}$, at a minimum, with observational data supporting a MAP $80-90 \mathrm{mmHg}$. MAP will be maintained $>80 \mathrm{mmHg}$, unless it compromises the hemodynamics. Vasoactive medications, inotropes or mechanical circulatory support may be needed.

Patients who remain comatose will have their neurological prognosis assessed per the ERC's and the European Society for Intensive Care Medicine recommendations. The multimodal neurological prognostication algorithm will be activated $>72 \mathrm{~h}$ after ROSC to avoid confounding from acute metabolic disturbances, sedation, analgesia and paralysis.

\section{Provisions for post-trial care $\{30\}$}

Patients will be followed for the duration of their hospitalization and up to 6 months after hospital discharge. At the time of hospital discharge, a research coordinator will determine each subject's modified Rankin scale (mRS) score and cerebral performance category (CPC). This will also occur at 6 months after hospital discharge via telephone call. In the case of injury or illness resulting from this research study, the subject's insurance company will be responsible for any costs resulting from underlying disease or treatments provided outside of this research study.

Outcomes $\{12\}$

Primary efficacy outcome

- Clinically-diagnosed EOP occurring < 4 days after initiation of mechanical ventilation

There are no validated diagnostic criteria for pneumonia in comatose OHCA survivors or those receiving some form of temperature modulation. Consequently, the definition of clinical pneumonia was adapted from diagnostic criteria for nosocomial pneumonia proposed by the Centers for Disease Control and Prevention/ National Healthcare Safety Network, American Thoracic Society and the Infectious Diseases Society of America, and published definitions of pneumonia in this patient population [18,23,24].
During the 72-h TTM period, clinical pneumonia will be defined as:

New or progressive lung infiltrate and at least one of the following ( $\mathrm{a}$ or $\mathrm{b})$ :

a. New purulent secretions or change in quantity or quality of sputum

b. Worsening gas exchange defined as (any of i through iii):

i. Oxygen desaturations or $\mathrm{PaO}_{2} / \mathrm{FIO}_{2} \leq 240$

ii. Increased oxygen requirements

1. Increase in daily minimum $\mathrm{FiO} 2$ of $\geq 0.20$ (20 points) over the daily minimum $\mathrm{FiO}_{2}$ of the first day in the baseline period, sustained for $\geq 2$ calendar days. Daily minimum defined by lowest value of $\mathrm{FiO}_{2}$ during a calendar day that is maintained for $>1 \mathrm{~h}$.

iii. Increased mechanical ventilator demand

1. A sustained increase in the daily minimum PEEP of $\geq 3 \mathrm{cmH}_{2} \mathrm{O}$ following a period of stability or improvement on the ventilator. PEEP values from 0 to $5 \mathrm{cmH} 2 \mathrm{O}$ are considered equivalent. Daily minimum defined by lowest value of PEEP during a calendar day that is maintained for $>1 \mathrm{~h}$.

After the 72-h TTM period, clinical pneumonia will be defined as:

New or progressive lung infiltrate and at least two of the following (a through e):

a. New purulent secretions or change in quantity or quality of sputum

b. Worsening gas exchange, not otherwise explained, defined as (any of i through iii):

i. Oxygen desaturations or $\mathrm{PaO}_{2} / \mathrm{FIO}_{2} \leq 240$

ii. Increased oxygen requirements

iii. Increased ventilator demand

c. Cough, dyspnea, tachypnea, rales, or bronchial breath sounds

d. Body temperature $>38^{\circ} \mathrm{C}$

e. Leukopenia $\left(<4000\right.$ white blood cells $\left./ \mathrm{mm}^{3}\right)$ or leukocytosis ( $>12,000$ white blood cells $/ \mathrm{mm}^{3}$ )

\section{Secondary outcomes}

A clinical diagnosis of pneumonia will be microbiologically confirmed using flexible fiberoptic bronchoscopy with $>10^{4}$ colony forming units (CFU) per $\mathrm{mL}$ of pathogenic bacteria or unprotected mini-bronchoalveolar lavage with $>10^{4} \mathrm{CFU} /$ $\mathrm{mL}$ of pathogenic bacteria in intubated patients [23, 24]. In non-intubated patients, an expectorated sputum sample will be used. Blinded, board certified pulmonologists will adjudicate any clinical or microbiological pneumonia diagnosis. 
- Microbiologically-confirmed EOP occurring < 4 days after initiation of mechanical ventilation

- Microbiologically-confirmed late-onset pneumonia occurring $\geq 4$ days after initiation of mechanical ventilation

- Clinically-diagnosed late-onset pneumonia occurring $\geq 4$ days after initiation of mechanical ventilation

- Incidence of non-pulmonary infections

- ICU-free days in the first 28 days of admission

- Mechanical ventilator-free days in the first 28 days of admission

- ICU LOS

- Hospital LOS

- ICU mortality

- Hospital mortality

- Discharge disposition

- Functional outcome at hospital discharge and 6 months after hospital discharge

- Good functional outcome will $\mathrm{mRS} \leq 0-3$ of or a CPC 1-2

- Functional outcome will be assessed by a research coordinator at hospital discharge and via telephone at 6 months

\section{Safety outcomes}

- Clostridioides difficile-associated diarrhea [25]

- Diagnosed according to the 2017 Infectious Diseases Society of American Clinical Practice Guidelines for Clostridium difficile Infection in Adults:

- Unexplained and new-onset $\geq 3$ unformed stools in $24 \mathrm{~h}$

- Positive Clostridioides difficile toxin gene polymerase chain reaction assay

- Type one (immediate-type) hypersensitivity reactions [26]

- Acute symptom onset (minutes to hours) involving the skin, mucosal tissue, or both (e.g., generalized hives, pruritus or flushing, swollen lips-tongue-uvula) and at least one of the following (a through c):

a. Respiratory compromise

b. Reduced blood pressure

c. Signs of end-organ dysfunction

- Gallbladder disease [20]

- Diagnosed sonographically as an echo without acoustical shadowing suggesting sludge and the presence of ceftriaxone-calcium salt

\section{Participant timeline $\{13\}$}

The sequence of events can be found in Table 3.

\section{Sample size $\{14\}$}

In the ANTHARTIC trial, the incidence of EOP at 5 days was $17 \%$ in the prophylaxis group and $31 \%$ in controls (absolute difference of 14\%) [18]. In contrast to our protocol, when using their definition of EOP, the incidence at 7 days was $19 \%$ in the prophylaxis group and 34\% in controls (absolute difference of 15\%) [18].

In the TTM- 1 trial, pneumonia occurred in $52 \%$ of patients treated at $33^{\circ} \mathrm{C}$ and $46 \%$ in those treated at $36^{\circ} \mathrm{C}$ [21]. Pneumonia was not defined as early- or late-onset. In a post hoc analysis, the incidence of pneumonia in patients admitted to centers administering antibiotic prophylaxis was $41 \%$ compared to $54 \%$ at centers that did not [27]. Clustering within centers was possible.

Using data from the International Cardiac Arrest Registry (INTCAR), the incidence of pneumonia, which was not defined as early- or late-onset, was 33\% with antibiotic prophylaxis [28]. Accordingly, 60 patients will be randomized to each group ( $n=120$ total). This provides $80 \%$ power at a two-tailed $5 \%$ level of significance to detect an absolute reduction in the incidence of EOP by $25 \%$, from 55 to $30 \%$.

\section{Recruitment $\{15\}$}

The study team is notified via electronic mail when a patient has orders for temperature management following OHCA. Additionally, the study team is alerted by a medical communications technician when a patient is being transported by emergency medical services to the hospital after an OHCA. In the event of slow recruitment, a second enrolling center may be activated following IRB approval.

\section{Assignment of interventions: allocation Sequence generation $\{16 \mathrm{a}\}$}

A computer-generated allocation sequence will randomize patients in a 1:1 ratio in blocks of six. Randomization will not be stratified.

\section{Concealment mechanism $\{16 \mathrm{~b}\}$}

The clinical trials pharmacist will be responsible for randomizing patients independent of study investigators. The clinical trials pharmacist will prepare the study drug for delivery to the bedside nurse for administration.

\section{Implementation $\{16 \mathrm{c}\}$}

A random order sequence was developed by the lead statistician. Patients will be enrolled by research team members, including physicians and pharmacists, as delineated in the Delegation of Authority log. The clinical trials pharmacist with dispense study drug according to the allocation sequence. 
Table 3 Sequence of events in the PROTECT trial

\begin{tabular}{|c|c|c|c|c|c|c|c|c|c|}
\hline & $\begin{array}{l}\text { By } 6 \mathrm{~h} \text { of ICU } \\
\text { admission }\end{array}$ & $\begin{array}{l}\text { Day } 1 \\
(0-24 \mathrm{~h})\end{array}$ & $\begin{array}{l}\text { Day } 2 \\
(24-48 \mathrm{~h})\end{array}$ & $\begin{array}{l}\text { Day } 3 \\
(48-72 \text { h) }\end{array}$ & $\begin{array}{l}\text { Day } 4 \\
\text { (72-96 h) }\end{array}$ & $\begin{array}{l}\text { Day } 7 \\
(168 \mathrm{~h})\end{array}$ & $\begin{array}{l}\text { Day } 1 \text { to hospital } \\
\text { discharge }\end{array}$ & $\begin{array}{l}\text { Hospital } \\
\text { discharge }\end{array}$ & $\begin{array}{l}\text { 6-month } \\
\text { follow-up }\end{array}$ \\
\hline Screening/enrollment & $x$ & & & & & & & & \\
\hline Study drug & & $x$ & $x$ & $x$ & & & & & \\
\hline Sputum culture & $x$ & & & & $x$ & & & & \\
\hline Rectal swab & $x$ & & & & $x$ & $x$ & & & \\
\hline Blood sample & $x$ & $x$ & & $x$ & & & & & \\
\hline EOP & & $x$ & $x$ & $x$ & & & & & \\
\hline Non-pulm. infection & & & & & & & $x$ & & \\
\hline ICU LOS & & & & & & & $x$ & & \\
\hline Ventilator-free days & & & & & & & $x$ & & \\
\hline Hospital LOS & & & & & & & $x$ & & \\
\hline ICU mortality & & & & & & & $x$ & & \\
\hline Hospital mortality & & & & & & & $x$ & & \\
\hline Discharge disposition & & & & & & & $x$ & & \\
\hline CDAD & & & & & & & $x$ & & \\
\hline Type-one allergy & & $x$ & $x$ & $x$ & & & & & \\
\hline Gallbladder disease & & & & & & & $x$ & & \\
\hline $\mathrm{mRS}$ & & & & & & & & $x$ & $x$ \\
\hline CPC & & & & & & & & $x$ & $x$ \\
\hline
\end{tabular}

CDAD Clostridioides difficile-associated diarrhea, EOP early-onset pneumonia, ICU intensive care unit, LOS length of stay, mRS modified Rankin scale, CPC cerebral performance category

\section{Assignment of interventions: blinding} Who will be blinded? $\{17 \mathrm{a}\}$

The PROTECT trial is quadruple-blind including the patient/LAR, bedside treatment team, research team, and outcome assessors (i.e., pneumonia adjudicators and functional outcome assessor). Study drug will be dispensed with an amber cover to mask the yellow tint to reconstituted and diluted ceftriaxone.

\section{Procedure for unblinding if needed $\{17 b\}$}

Unblinding may occur for a serious adverse event or if the bedside treatment team determines it is medically necessary. Results of unblinding will not be communicated to study investigators, members of the pneumonia adjudication committee, or the outcome evaluators. Accidental unblinding will be dealt with on a case-by-case basis and patients may continue in the study depending on the circumstance.

\section{Data collection and management}

\section{Plans for assessment and collection of outcomes $\{18 \mathrm{a}\}$}

Data collection forms will be completed for patients who are randomized. Data will be transcribed into REDCap, which is a secure, web-based application for data capture. Screening logs will be maintained and reasons for exclusion will be recorded. Data collected may follow the International Liaison Committee on Resuscitation's
Utstein criteria for OHCA. Data will be collected by clinical research coordinators or study investigators from medical records, family members, ambulance run reports, or other sources.

\section{Plans to Promote Participant Retention and Complete Follow-up $\{18 b\}$}

Patients will be followed for the duration of their hospitalization and up to 6 months after hospital discharge. At the time of hospital discharge, a research coordinator will determine each patient's mRS score and CPC. This will also occur at 6 months after hospital discharge via telephone call.

\section{Data management $\{19\}$}

Data will be transcribed into REDCap, which is a secure, web-based application for data capture. Screening logs will also be maintained in REDCap and reasons for exclusion will be recorded. Upon data exportation, a random PROTECT trial identification number will be assigned. Data entered into the REDCap database will include checks for value ranges and empty fields.

\section{Confidentiality $\{27\}$}

Documents will be retained at Maine Medical Center for 15 years, which is in compliance with the United States' Food and Drug Administration Code of Federal Regulations (21 
CFR \$312.62[c]). Access to source documents may be permitted for trial-related monitoring and audits, when appropriate. Individual data for monitoring, carrying out quality control, and auditing biomedical research may be shared at the discretion of the study investigators.

Plans for collection, laboratory evaluation, and storage of biological specimens for genetic or molecular analysis in this trial/future use $\{33\}$

\section{Microbiome and resistome assessment}

Sputum and rectal swabs will be collected prior to study drug initiation, within $24 \mathrm{~h}$ of completing study drug, and on study-day seven or at the time of withdrawal of life sustaining therapies, whichever occurs first (rectal swab). Total nucleic acids will be extracted and resistomes determined by shotgun metagenomic sequencing using super high-throughput methods.

Total nucleic acids will be extracted from sputum and from rectal swabs using ZymoBIOMICS reagents (Zymo), and shotgun libraries will be prepared and include inline barcodes. Libraries will be pooled in groups of 96 for super high-throughput sequencing with paired-end reads (150 bp) using the Illumina HiSeq 4000 platform.

Library preparation and sequencing reads will be performed at the UC Davis Genome Center. Reads will be de-multiplexed by barcode and trimmed by ILLUMACLIP, and contaminating human sequence reads will be filtered. Remaining reads will be assembled into metagenomes using Velvet, and resistance genotypes will be identified and quantified using ResFams and ShortBRED, respectively [29-32].

\section{Inflammation assessment}

Peripheral blood samples $(10 \mathrm{~mL})$ will be obtained before study drug, and on study-day 1 and study-day 3 (30 mL total) after discontinuation of study drug. Subpopulations of white blood cells will be measured using flow cytometry. Mononuclear cells will be isolated using Ficoll-Paque $^{\mathrm{Tm}}$. Generation of adenosine by $\mathrm{T}$ lymphocyte will be measured using Malachite green phosphate assay (Sigma) [33]. Analysis of genes involved in $\mathrm{T}$ cell response will be performed with total RNA isolated from CD3+ lymphocytes using RNeasy Mini Kit (Qiagen). Gene expression will be analyzed using Oligo GEArray ${ }^{\circ}$ Human T-cell and B-cell Activation Microarray (SABiosciences, OHS-053).

Multi-parametric flow cytometric analysis will be conducted on whole blood cells before the study drug and on study-day 1 and study-day 3. Cells will be stained using fluorochrome-conjugated antibodies. For intracellular staining, cells will be permeabilized using BD Cytofix/Cytoperm ${ }^{\mathrm{TM}}$ buffer. The percentage of lymphocyte subsets will be determined within viable cell populations using the MACSQuant 10 analyzer. Myeloid cells (neutrophils and monocytes) will be analyzed to validate ceftriaxone's effect in lymphocytes.

\section{Statistical methods}

Statistical methods for primary and secondary outcomes \{20a\}

The primary efficacy endpoint (pneumonia within 4 days) will be analyzed on an intention-to-treat basis. We will use survival analysis techniques to evaluate the primary endpoint, with failure defined as incident pneumonia and failure time defined as the day on which pneumonia is first identified. Although follow-up time is short, survival analysis will allow us to account for the competing risk of death. Patients who die within 4 days will be censored at the day of death. Patients who do not die and do not develop pneumonia within 4 days will be censored at day 4 . We will use standard survival analysis techniques to visually examine differences in pneumonia incidence and will estimate the hazard ratio as our primary outcome. Twotailed tests of significance will be used, and $p \leq 0.05$ will be considered significant. Analyses will be performed using SAS version 9.4 and $R$ version 4.0.2.

The secondary efficacy endpoints will be analyzed on an intention-to-treat basis. Late-onset pneumonia and other infectious outcomes will be analyzed using the same statistical approach as the primary efficacy outcome. Mortality at day 28 will be analyzed using the chisquare test.

\section{Interim analyses $\{21 \mathrm{~b}\}$}

A single interim analysis will be conducted when 60 (50\%) subjects have been enrolled. The independent Data Safety Monitoring Board will be empowered to stop the trial under the following circumstances:

- Harm

- There is a statistically significant increased risk for serious adverse events, including Clostridium difficile-associated diarrhea, gallbladder toxicity, or type one hypersensitivity reactions, during the interim analysis of the first $n=60$ patients enrolled. The $p$ value threshold for harm will be set at 0.05 .

- Safety

- Significant safety concerns emerge and the Data Safety Monitoring Board (DSMB) and IRB choose to pause or stop the trial.

The trial will not be terminated for given the intermediate outcome of interest is pneumonia, rather than death or other concrete outcomes (e.g., stroke, myocardial infarction). Additionally, it will not allow for a complete assessment of the microbiome or inflammation. 
Methods for additional analyses (e.g., subgroup analyses) $\{20 \mathrm{~b}\}$

Abundance of antibiotic resistance-associated genotypes in resistomes will be compared within and between treatment groups. If ceftriaxone or placebo alters resistomes, ANOVA will be performed across genotypes. The abundance of each genotype will be compared between ceftriaxone- and placebo-treated patients post-intervention by both Student's $T$ test and $\chi^{2}$ to establish direct changes associated with ceftriaxone and whether those deviate from potential changes in resistomes due to ICU admission. All statistical analyses will be performed using GraphPad Prism 8 (GraphPad Software Inc).

We expect to obtain $56.3 \pm 4.5 \times 10^{6}$ of total white blood cells, containing $14.7 \pm 1.7 \times 10^{6}$ mononuclear cells and 6.3 $\pm 1.1 \times 10^{6}$ of CD3+ T cells from $10 \mathrm{~mL}$ of blood. We will use approximately $1 \times 10^{6} \mathrm{CD} 3+\mathrm{T}$ cells to determine direct effects of ceftriaxone on CD73 expression and adenosine generation. We will use approximately $3 \times 10^{6}$ of $\mathrm{CD} 3+\mathrm{T}$ cells to isolate mRNA for gene expression analysis after treatment with ceftriaxone. Approximately $2-3 \times 10^{6}$ whole blood cells will be used for flow cytometric analysis. Statistical analysis will be performed using the GraphPad Prism 7.0 software (GraphPad Software Inc). Comparisons between groups will be performed using two-tailed unpaired $t$ tests. Comparisons between several groups will be performed using one-way ANOVA followed by appropriate post hoc tests.

\section{Methods in analysis to handle protocol non-adherence} and any statistical methods to handle missing data $\{20 \mathrm{c}\}$ Missing data will be reported at the time of publication. If further statistical analyses reveal substantial missing data, multiple imputation will be considered.

Plans to give access to the full protocol, participant-level data, and statistical code $\{31 \mathrm{c}\}$

The full trial protocol will be published as a supplement along with the results of the primary and secondary analyses in a single publication. Participant-level data will be made available according to the National Institutes of Health's Policy for Data Management and Sharing. Data will be made accessible as soon as possible, and no later than the time of an associated publication, or the end of the trial, whichever comes first.

\section{Oversight and monitoring}

Composition of the coordinating center and trial steering committee $\{5 \mathrm{~d}\}$

Maine Medical Center is located in Portland, Maine, USA and has 637 licensed beds including a 12-bed cardiac ICU and 32-bed mixed medical, surgical, and neurological ICU. Its catchment area includes the state of Maine and northeast New Hampshire including approximately 1.3 million people. The Neurocritical Care team has around-the-clock coverage by an attending physician and an advanced practice provider (i.e., Physician Assistant or Nurse Practitioner).

The inflammation analysis will be completed at Maine Medical Center Research Institute in Scarborough, Maine, USA. The microbiome analyses will be done at the University of New England, Biddeford, Maine, USA, with library preparation and sequencing reads performed at the UC Davis Genome Center in Davis, CA, USA.

A Clinical Trial Steering Committee consisting of the principal investigator and co-investigators will oversee the trial. Weekly Neurocritical Care research meetings will be used to discuss enrollment and safety issues. A pneumonia adjudication board will review each diagnosis of pneumonia in a blinded manner. A statistician and data analyst from the Maine Medical Center Research Institute will analyze the data in a blinded manner for the interim analysis and final analysis.

\section{Composition of the data monitoring committee, its role and reporting structure $\{21 \mathrm{a}\}$}

A independent DSMB will execute a Data Safety Monitoring Plan (DSMP) and be chaired by a physician. The DSMB will consist of a board certified infectious diseases physician, board certified pulmonologist, and a senior biostatistician. The DSMB will meet at least every 4 months and more frequently during the interim analysis. More information on the DSMB and DSMP can be found in the trial protocol.

\section{Adverse event reporting and harms \{22\}}

Reportable serious adverse events will be reported to the IRB and DSMB within five business days of the principal investigator notification. Reportable serious adverse events resulting in death will be reported to the IRB and DSMB within $48 \mathrm{~h}$ of the principal investigator notification. Unexpected serious adverse events deemed reasonably or definitely associated with the study drug will be reported to the IRB and DSMB within five business days of the principal investigator notification. Approximately $60 \%$ of patients who survive to the hospital after out-of-hospital cardiac arrest will survive. Death (other than as noted above) will be a serious adverse event and reported to the IRB and DSMB within five business days.

\section{Frequency and plans for auditing trial conduct $\{23\}$}

As described in the "Strategies to Improve Adherence to Interventions $\{11 c\}$ " section, a clinical research associate appointed by Maine Medical Center's Institutional Review Board (IRB) may visit during the study to ensure proper conduct. Additionally, the DSMB will be 
empowered to share information related to study conduct with the Clinical Trial Steering Committee and the IRB. Information shared should focus on trial procedures, and may include the following:

- Rates of recruitment, ineligibility, noncompliance, protocol violations and dropouts

- Completeness and timeliness of data

- Degree of concordance between site evaluation of events and centralized review

- Balance between study arms on important prognostic variables

- Accrual within important subsets

\section{Plans for communicating important protocol} amendments to relevant parties $\{25\}$

Protocol amendments will be approved by the Maine Medical Center IRB. Protocol training will occur after any protocol amendment. The funder, National Institute of General Medical Sciences, will be notified of the first and last enrollment. The ClinicalTrials.gov listing will be updated periodically for enrollment or major protocols changes.

\section{Dissemination plans $\{31 \mathrm{a}\}$}

Analyses will be performed 6 months after hospital discharge of the last patient. The full-length manuscript will be submitted to a peer-reviewed international medical journal. Authorship will follow the guidelines set forth by the International Committee of Medical Journal Editors. The main publication will include the primary and secondary efficacy outcomes, safety outcomes, inflammation data, and microbiome data. Subsequent publications may be considered by the study investigators at the conclusion of the trial.

\section{Discussion}

This manuscript describes the design and rationale for the PROTECT trial. The short-term objectives of the trial are to (1) assess the impact of prophylactic ceftriaxone on the incidence of EOP occurring $<4$ days after initiation of mechanical ventilation in comatose survivors of OHCA, (2) quantify the ceftriaxone's effect on T cellmediated inflammation, (3) determine if prophylactic ceftriaxone alters the innate microbiome and resistome, and (4) collect preliminary data needed to design a definitive trial powered for functional outcome or mortality. The long-term objective is to conduct a large, multicenter trial examining functional outcome and mortality.

The ANTHARTIC trial reported a $47 \%$ reduction in EOP when intravenous amoxicillin-clavulanate was administered prophylactically for 2 days following OHCA. ${ }^{18}$ Our study will extend ANTHARTIC's findings in several critical ways: (1) inclusion of OHCA survivors with all initial heart rhythms, (2) use of a lower-risk antibiotic available in the USA that has not previously been tested after OHCA, (3) study of the anti-inflammatory effects of ceftriaxone to determine a mechanism by which it improves clinical outcomes, and (4) complete metagenomic assessment of bacterial resistomes pre- and post-ceftriaxone prophylaxis.

In a rat model, ceftriaxone reduced IFN- $\gamma$ and TNF- $\alpha$ in the injured parietal cortex and improved learning and spatial memory function [34]. Ceftriaxone also reduced IFN- $\gamma$ and IL-17 secretion in a mouse model of multiple sclerosis by altering antigen presentation and activation of myelin-specific $\mathrm{T}$ lymphocytes [35]. Other studies have shown ceftriaxone dampens excitotoxicity by decreasing glutamatergic activity [34, 36-39]. Excitotoxicity may be a mechanism of injury following OHCA, so ceftriaxone's ability to attenuate this response is promising [40].

$\mathrm{T}$ cells promote neuroinflammation and neuronal cell death via IFN- $\gamma$ and TNF- $\alpha$ [40-43]. We found incubation of human CD3+ T cells with ceftriaxone for $24 \mathrm{~h}$ increased expression of $\mathrm{CD} 73$, but not the number of cells expressing CD73 (Fig. 1). However, cell surface expression of CD73 (non-permeabilized cells) was increased on $\mathrm{T}$ cells treated with ceftriaxone as defined by shift towards higher immunofluorescence. Expression of CD73 was increased at the cell surface of both CD8 and CD4 positive $\mathrm{CD} 3 \mathrm{~T}$ lymphocytes. $\mathrm{CD} 73$ is an adenosinegenerating enzyme, and adenosine has potent immunosuppressive and anti-inflammatory effects in $\mathrm{T}$ cells. Consistent with this concept, we found that the level of CD73 inversely correlated with IFN- $\gamma$ levels in both CD4+ and CD8+ T cells.

The effects of prophylactic antibiotics on bacterial resistance after OHCA are not known, but antibiotic resistance is a global concern [44]. We will assess emergence of resistant bacteria in both treatment and control patients. Control patients may be exposed to longer courses of broadspectrum antibiotics due to higher rates of pneumonia. We will create data on drug concentrations in stool, before-andafter effects of ceftriaxone on expression of resistance genes, and on richness, diversity, and relative abundance of taxa.

Earlier studies in acutely brain-injured patients found antibiotic prophylaxis did not induce bacterial resistance using limited analyses [15-17]. The ANTHARTIC trial evaluated intestinal acquisition of multidrug-resistant bacteria on day 7 on solid selective media using stool samples, limiting their observations to conditionally cultivatable organisms [18]. No difference was found following 2 days of amoxicillin-clavulanate administration compared to placebo. The gold standard for evaluating bacterial resistance is resistome analysis with high-throughput sequencing, which will be utilized in our trial. 


\section{Conclusions}

The PROTECT trial is a phase II trial of prophylactic ceftriaxone for 3 days in comatose OHCA survivors. The trial will provide preliminary data needed for a definitive phase III trial, as well as novel data on ceftriaxone's antiinflammatory effects and its impact on the microbiome. Our ultimate goal is to improve survival and quality of life in survivors of OHCA.

\section{Trial status}

This manuscript describes the PROTECT trial protocol version \#3 dated 6/11/21. Recruitment will begin in August 2020 and the anticipated completion is August 2023.

\section{Abbreviations \\ ANTHARTIC: Antibiotherapy during Therapeutic Hypothermia to Prevent Infectious Complications; CPC: Cerebral performance category; CPR: Cardiopulmonary resuscitation; EOP: Early-onset pneumonia; DSMB: Data Safety Monitoring Board; DSMP: Data Safety Monitoring Plan; EFIC: Exception from Informed Consent; INTCAR: International Cardiac Arrest Registry; IRB: Institutional Review Board; LAR: Legally authorized representative; mRS: Modified Rankin scale; OHCA: Out-of-hospital cardiac arrest; PROTECT: Ceftriaxone to PRevent pneumOnia and inflammaTion aftEr Cardiac arresT; ROSC: Return of spontaneous circulation; TTM: Targeted temperature management}

\section{Acknowledgements}

The authors would like to thank the members of the greater Portland, ME, USA community for their engagement in the EFIC process. We appreciate the work done at the Center for Anti-infective Research and Development at Hartford Hospital and the Antimicrobial Stewardship Program at Maine Medical Center for their development of susceptibility and cumulative fraction of response data. Finally, we would like to thank the members of the Administrative Core and the Community Engagement Bioethics and Outreach Core, as well as the many others who enabled us to conduct this trial.

\section{Authors' contributions $\{31 \mathrm{~b}\}$}

DJG is the Principal Investigator, designed the trial, and wrote the manuscript; SVR is a Co-investigator, designed the trial, and wrote the manuscript; MAM is a Co-investigator, designed the trial, and wrote the manuscript; RRR is a Co-investigator and designed the trial; BG is a Co-investigator and designed the trial; TLM is a Co-investigator and designed the trial; SB is a Clinical Research Coordinator; JTD designed the trial and will complete the inflammation assessment; AE is a Clinical Research Coordinator; TV is a member of the Pneumonia Adjudication Committee; PL is a Sub-investigator and wrote the manuscript; CL is a Clinical Research Coordinator; FLL is a Subinvestigator and lead statistician; PM is a Sub-investigator and designed the trial; BM is a Clinical Research Coordinator; MS is a Clinical Research Coordinator; JW is a member of the Pneumonia Adjudication Committee; JZ designed the trial and wrote the manuscript; DS is the lead investigator on the grant and wrote the manuscript; DBS is a Co-investigator, designed the trial, and wrote the manuscript. The authors read and approved the final manuscript.

\section{Funding $\{4\}$}

The trial is supported by the National Institute of General Medical Sciences (1P20GM139745-01). The funding agency was not involved with study design, data collection, analysis and interpretation of data, writing of the manuscript, or the decision to submit the manuscript for publication. DJG, SVR, MAM, RRR, TM, CL, AE, MS, BM, SB, LL, DW, and DBS are supported by this grant.

\section{Availability of data and materials $\{29\}$}

The data that support the findings of this study are available from National Institute of General Medical Sciences but restrictions apply to the availability of these data, which were used under license for the current study, and so are not publicly available. Data are however available from the authors upon reasonable request and with permission of National Institute of General Medical Sciences.

\section{Declarations}

Ethics approval and consent to participate $\{24\}$

This trial was approved by the Maine Medical Center Institutional Review Board (1661144-6).Informed consent to participate will be obtained from all subjects or their LAR in writing or electronically using DocuSign.

\section{Consent for publication $\{32\}$}

All subjects enrolled in the trial will consent to publication as part of the EFIC or informed consent process using an institutional informed consent form approved by the Maine Medical Center Institutional Review Board.

\section{Competing interests $\{\mathbf{2 8}\}$}

The authors declare that they have no competing interests.

\section{Author details}

${ }^{1}$ Department of Pharmacy, Maine Medical Center, Portland, ME, USA. ${ }^{2}$ Maine Medical Center Research Institute, Scarborough, ME, USA. ${ }^{3}$ Tufts University School of Medicine, Boston, MA, USA. ${ }^{4}$ University of New England College of Osteopathic Medicine, Biddeford, ME, USA. ${ }^{5}$ Department of Critical Care Services, Maine Medical Center, Portland, ME, USA. ${ }^{6}$ Maine Medical Partners, MaineHealth Cardiology, Scarborough, ME, USA. ${ }^{7}$ Maine Medical Center Neuroscience Institute, Maine Medical Center, Portland, ME, USA.

Received: 10 August 2021 Accepted: 23 February 2022

Published online: 04 March 2022

\section{References}

1. Rello J, Emili D. Pneumonia in the intensive care unit. Crit Care Med. 2003; 31:2544-51.

2. Perbet S, Mongardon N, Dumas F, et al. Early-onset pneumonia after cardiac arrest: characteristics, risk factors and influence on prognosis. Am J Respir Crit Care Med. 2011;184:1048-54.

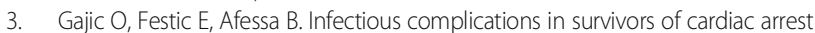
admitted to the medical intensive care unit. Resuscitation. 2004;60:65-9.

4. Rello J, Vallés J, Jubert $P$, et al. Lower respiratory tract infections following cardiac arrest and cardiopulmonary resuscitation. Clin Infect Dis. 1995;21: 310-4.

5. Gaussorgues P, Gueugniaud PY, Vedrinne JM, Salord F, Mercatello A, Robert D. Bacteremia following cardiac arrest and cardiopulmonary resuscitation. Intensive Care Med. 1988;14:575-7.

6. Kim YM, Youn CS, Kim SH, et al. Adverse events associated with poor neurological outcome during targeted temperature management and advanced critical care after out-of-hospital cardiac arrest. Crit Care. 2015;19:283.

7. Bjork RJ, Snyder BD, Campion BC, Loewenson RB. Medical complications of cardiopulmonary arrest. Arch Intern Med. 1982;142:500-3.

8. Voorhees WD, Babbs CF, Tacker WA Jr. Regional blood flow during cardiopulmonary resuscitation in dogs. Crit Care Med. 1980;8(3):134-6.

9. Grimaldi D, Guivarch E, Neveux N, et al. Markers of intestinal injury are associated with endotoxemia in successfully resuscitated patients. Resuscitation. 2013;84(1):60-5.

10. Grimaldi D, Sauneuf B, Guivarch E, et al. High level of endotoxemia following out-of-hospital cardiac arrest is associated with severity and duration of postcardiac arrest shock. Crit Care Med. 2015;43:2597-04.

11. Adrie C, Laurent I, Monchi M, Cariou A, Dhainaou JF, Spaulding C. Postresuscitation disease after cardiac arrest: a sepsis-like syndrome? Curr Opin Crit Care. 2004;10:208-12

12. Adrie C, Adib-Conquy M, Laurent I, et al. Successful cardiopulmonary resuscitation after cardiac arrest as a "sepsis-like" syndrome. Circulation. 2002;106:562-8.

13. Soppi E, Lindroos M, Nikoskelainen J, Kalliomäki JL. Effect of cardiopulmonary resuscitation-induced stress on cell-mediated immunity. Intensive Care Med. 1984;10:287-92.

14. Beurskens CJ, Horn J, de Boer AM, Schultz MJ, van Leeuwen EM, Vroom MB, et al. Cardiac arrest patients have an impaired immune response, which is not influenced by induced hypothermia. Crit Care. 2014;18:R162. 
15. Sirvent JM, Torres A, El-Ebiary M, Castro P, de Batlle J, Bonet A. Protective effect of intravenously administered cefuroxime against nosocomial pneumonia in patients with structural coma. Am J Respir Crit Care Med. 1997;155:1729-34

16. Acquarolo A, Urli T, Perone G, Giannotti C, Candiani A, Latronico N. Antibiotic prophylaxis of early onset pneumonia in critically ill comatose patients. A randomized study. Intensive Care Med. 2005;31:510-6.

17. Vallés J, Peredo R, Burgueño MJ, et al. Efficacy of single-dose antibiotic against early-onset pneumonia in comatose patients who are ventilated. Chest. 2013;143:1219-25.

18. François B, Cariou A, Clere-Jehl R, et al. Prevention of early ventilatorassociated pneumonia after cardiac arrest. N Engl J Med. 2019;381:1831-42.

19. Guidance for Institutional Review Boards, Clinical Investigators, and Sponsors Exception from Informed Consent Requirements for Emergency Research (2013). Food and Drug Administration. https://www.fda.gov/regula tory-information/search-fda-guidance-documents/exception-informedconsent-requirements-emergency-research.

20. Ceftriaxone [package insert]. Weston, FL: Apotex Corporation; 2011.

21. Nielsen N, Wetterslev J, Cronberg T, et al. Targeted temperature management at $33^{\circ} \mathrm{C}$ vs. $36^{\circ} \mathrm{C}$ after cardiac arrest. N Engl J Med. 2013;369: 2197-206.

22. Dankiewicz J, Cronberg T, Lilja G, et al. Hypothermia versus normothermia after out-of-hospital cardiac arrest. N Engl J Med. 2021;384:2283-94.

23. Skrupky LP, McConnell K, Dallas J, Kollef MH. A comparison of ventilatorassociated pneumonia rates as identified according to the National Healthcare Safety Network and American College of Chest Physicians Criteria. Crit Care Med. 2012;40:281-4.

24. American Thoracic Society; Infectious Diseases Society of America. Guidelines for the management of adults with hospital-acquired, ventilatorassociated, and healthcare-associated pneumonia. Am J Respir Crit Care Med. 2005;171:388-416.

25. McDonald CL, Gerding DN, Johnson S, et al. Clinical Practice Guidelines for Clostridium difficile Infection in Adults and Children: 2017 Update by the Infectious Diseases Society of America (IDSA) and Society for Healthcare Epidemiology of America (SHEA). Clin Infect Dis. 2018;66:e1-e48.

26. Sampson HA, Muñoz-Furlong A, Campbell RL, et al. Second symposium on the definition and management of anaphylaxis: summary report--Second National Institute of Allergy and Infectious Disease/Food Allergy and Anaphylaxis Network symposium. J Allergy Clin Immunol. 2006;117:391-7.

27. Harmon MBA, Hodiamont CJ, Dankiewicz J, et al. Microbiological profile of nosocomial infections following cardiac arrest: Insights from the targeted temperature management (TTM) trial. Resuscitation. 2020;148:227-33.

28. The International Cardiac Arrest Registry. Maine Medical Center Research institute, Scarborough, Maine. 2021. https://mmcri.org/?page_id=15952. Accessed May 3, 2021.

29. Russell JA, Epstein LG, Greer DM, et al. Brain death, the determination of brain death, and member guidance for brain death accommodation requests. Neurology. 2019;92:228-304

30. Zerbino DR, Birney E. Velvet: algorithms for de novo short read assembly using de Bruijn graphs. Genome Res. 2008;18:821-9.

31. Gibson MK, Forsberg KJ, Dantas G. Improved annotation of antibiotic resistance determinants reveals microbial resistomes cluster by ecology. ISME J. 2015:9:207-16.

32. Kaminski J, Gibson MK, Franzosa EA, et al. High-specificity targeted functional profiling in microbial communities with ShortBRED. PLoS Comput Biol. 2015;11:e1004557.

33. Ryzhov SV, Pickup MW, Chytil A, et al. Role of TGF-beta signaling in generation of CD39+CD73+ myeloid cells in tumors. J Immunol. 2014;193: 3155-64.

34. Wei J, Pan X, Pei Z, et al. The beta-lactam antibiotic, ceftriaxone, provides neuroprotective potential via anti-excitotoxicity and anti-inflammation response in a rat model of traumatic brain injury. The J Trauma Acute Care Surg. 2012;73:654-60.

35. Melzer N, Meuth SG, Torres-Salazr D, et al. A beta-lactam antibiotic dampens excitotoxic inflammatory CNS damage in a mouse model of multiple sclerosis. PLoS One. 2008;3:e3149.

36. Hameed MQ, Hsieh TH, Morales-Quezada L, et al. Ceftriaxone treatment preserves cortical inhibitory interneuron function via transient salvage of GLT-1 in a rat traumatic brain injury model. Cereb Cortex. 2019;29:4506-18.
37. Krzyzanowska W, Pomierny B, Bystrowska B, et al. Ceftriaxone- and Nacetylcysteine-induced brain tolerance to ischemia: Influence on glutamate levels in focal cerebral ischemia. PLoS One. 2017;12:e0186243.

38. Jagadapillai R, Mellen NM, Sachleben LR Jr, Gozal E. Ceftriaxone preserves glutamate transporters and prevents intermittent hypoxia-induced vulnerability to brain excitotoxic injury. PLoS One. 2014;9:e100230.

39. Hu YY, et al. Ceftriaxone modulates uptake activity of glial glutamate transporter-1 against global brain ischemia in rats. J Neurochem. 2015;132: 194-205.

40. Tai $\mathrm{CH}$, Bellesi $\mathrm{M}, \mathrm{Chen} \mathrm{AC}$, et al. A new avenue for treating neuronal diseases: Ceftriaxone, an old antibiotic demonstrating behavioral neuronal effects. Behav Brain Res. 2019:364:149-56.

41. Gonzalez H, Pacheco R. T-cell-mediated regulation of neuroinflammation involved in neurodegenerative diseases. Journal of Neuroinflammation. 2014;11:201.

42. Daglas M, Draxler DF, Ho H, et al. Activated CD8+ T cells cause long-term neurological impairment after traumatic brain injury in mice. Cell Reports. 2019;29:1178-1191.e1176.

43. Clemenzi MN, Wellhauser L, Aljghami ME, Belsham DD. Tumour necrosis factor a induces neuroinflammation and insulin resistance in immortalised hypothalamic neurones through independent pathways. J Neuroendocrinol. 2019;31:e12678

44. Van Puyvelde S, Deborggraeve S, Jacobs J. Why the antibiotic resistance crisis requires a One Health approach. Lancet Infect Dis. 2018;18:132-4.

\section{Publisher's Note}

Springer Nature remains neutral with regard to jurisdictional claims in published maps and institutional affiliations.
Ready to submit your research? Choose BMC and benefit from:

- fast, convenient online submission

- thorough peer review by experienced researchers in your field

- rapid publication on acceptance

- support for research data, including large and complex data types

- gold Open Access which fosters wider collaboration and increased citations

- maximum visibility for your research: over $100 \mathrm{M}$ website views per year

At BMC, research is always in progress.

Learn more biomedcentral.com/submissions 